\title{
Impact of Oxygen Delivery Patterns on Arterial Oxygen Saturation Among Children
}

\author{
Deepali Gunjal $^{\mathrm{a}, \mathrm{d}}$, Radha Vaidyanathan ${ }^{\mathrm{b}}$, Vaibhav Bhosale ${ }^{\mathrm{c}}$
}

\begin{abstract}
Background: Adequate oxygenation to treat hypoxia by suitable oxygen delivery pattern is the essence of pediatric critical care medicine. Monitoring oxygen saturation $\left(\mathrm{SpO}_{2}\right)$ is thus essential in order to decide course of treatment. It is commonly carried out using painless sequential non-invasive pulse oximetry as well by severely painful random arterial blood gas (ABG) analysis.
\end{abstract}

Methods: This study compared both methods to determine the impact of various oxygen delivery patterns upon $\mathrm{SpO}_{2}$ at bedside for meaningful and continuous evaluation. In a prospective cohort adopted for study, 60 children from acute pediatric wards of an academic tertiary referral hospital were observed. Children were eligible for enrollment using cross sectional approach, if the treating pediatricians ordered an $\mathrm{ABG}$, on pulse oximetry monitoring with oxygen support by any oxygen delivery device using a validated checklist.

Results: Among all the samples, $60 \%$ were infants and $55 \%$ were male. Complaints on admission were vomiting (35\%), shortness of breath $(33 \%)$, diarrhea $(28 \%)$, lethargy (31\%) and Kussmaul breathing $(30 \%)$. $\mathrm{SpO}_{2}$ was $>90 \%$ for $88 \%$ of children. Oxy hood box was used to deliver oxygen to $73 \%$ of children. $\mathrm{SpO}_{2}$ had positive correlation with oxygen delivery methods $(\mathrm{r}=0.8)$ and partial pressure of oxygen $\left(\mathrm{PaO}_{2}\right)$ by $\mathrm{ABG}$ analysis had positive correlation with $\mathrm{SpO}_{2}$ by pulse oximetry $(r=0.9)$.

Conclusions: Pulse oximetry can be used as a reliable bed side tool to evaluate oxygen delivery patterns and diagnose early respiratory failure in emergency settings where ABG analysis facility is not available.

Keywords: ABG analysis; Arterial oxygen saturation; Oxygen delivery patterns

Manuscript submitted June 22, 2020, accepted July 10, 2020

Published online July 30, 2020

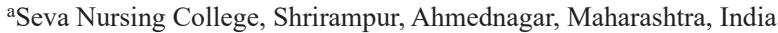

${ }^{\mathrm{b}}$ Child Health, Research and Hospital Management, Trichy, Tamilnadu, India

'Pravara Institute of Medical Sciences, College of Nursing, Loni Bk, Ahmednagar, Maharashtra, India

${ }^{\mathrm{d} C o r r e s p o n d i n g ~ A u t h o r: ~ D e e p a l i ~ G u n j a l, ~ S e v a ~ N u r s i n g ~ C o l l e g e, ~ S h r i r a m p u r, ~}$ Ahmednagar, Maharashtra, 413 736, India.

Email: deepugunja1777@gmail.com

doi: https://doi.org/10.14740/ijcp388

\section{Introduction}

Oxygen therapy plays a major role for respiratory care to treat hypoxemia in pediatric critical care medicine. Oxygen delivery patterns have devices that vary from a simple nasal cannula to sophisticated humidified systems. Several methods of noninvasive oxygen delivery patterns for children include head box oxygen (oxy hood), free flow oxygen source held close to infants' nose, facemask, nasal prongs, nasal catheter and nasopharyngeal catheter [1]. Undoubtedly, oxygen therapy is an important tool that has saved many lives and improved others [2]. Monitoring oxygen saturation $\left(\mathrm{SpO}_{2}\right)$ is thus essential in order to decide course of treatment. It is commonly carried out using painless sequential non-invasive pulse oximetry as well by severely painful random arterial blood gas $(\mathrm{ABG})$ analysis. Pulse oximetry is a monitoring method that indirectly determines oxygenation adequacy in a non-invasive manner [3]. ABG analysis checks how well lungs are able to move oxygen into blood and remove carbon dioxide from blood which is an invasive method [4]. ABG analysis is helpful to assess effectiveness of ventilation, circulation and perfusion especially in critically ill children.

Among 334 million people who suffer from asthma globally, $14 \%$ are children with chronic diseases of childhood including respiratory disorders. In India among 26.3 million cases, the incidence rate of respiratory disorders in children is about 2,173 cases per lakh who at some point of treatment require oxygen or ventilation. In order to assess oxygenation correctly, an understanding of the $\mathrm{ABG}$ values, relationship between partial pressure of oxygen $\left(\mathrm{PaO}_{2}\right)$ and $\mathrm{SpO}_{2}$ are required [5].

$\mathrm{ABG}$ analysis provide a snapshot of acid base balance by measuring hydrogen ion concentration in blood $(\mathrm{pH})$, oxygen saturation $\left(\mathrm{SaO}_{2}\right), \mathrm{PaO}_{2}$, partial pressure of carbon dioxide $\left(\mathrm{PaCO}_{2}\right)$, concentration of bicarbonate $\left(\mathrm{HCO}_{3}{ }^{-}\right)$and base excess in arterial blood. In addition, it indicates the primary source of disturbance (i.e., respiratory or metabolic) in homeostasis and how effectively patient's body is compensating for the acid base disturbance and whether the patient's total blood volume is adequate for transporting all nutrients that the body's tissues require [6].

However, $A B G$ is a painful and expensive test. One ABG typically leads to a cascade of ABGs, multiplying costs and blood loss. ABGs may be contaminated with venous blood as it requires expert skill to perform in children with very thin 
Table 1. Interpretation Guidelines for ABG Analysis Results

\begin{tabular}{|c|c|c|c|c|c|}
\hline $\begin{array}{l}\text { Component normal } \\
\text { range unit measure }\end{array}$ & pH:7.35 -7.45 & $\mathrm{PaO}_{2}: 80$ - 100 mm Hg & $\mathrm{PaCO}_{2}: 36-44 \mathrm{~mm} \mathrm{Hg}$ & $\mathrm{HCO}_{3}^{--}: 22-26 \mathrm{mmol} / \mathrm{L}$ & $\mathrm{K}^{+}: 3.5$ - $6 \mathrm{mmol} / \mathrm{L}$ \\
\hline Respiratory acidosis & Decrease & Decrease & Increase & Normal & Increase \\
\hline Respiratory alkalosis & Increase & Normal & Decrease & Normal & Decrease \\
\hline Metabolic acidosis & Decrease & Normal & Normal & Decrease & Increase \\
\hline Metabolic alkalosis & Increase & Normal & Normal & Increase & Decrease \\
\hline
\end{tabular}

ABG: arterial blood gas; $\mathrm{PaO}_{2}$ : partial pressure of oxygen; $\mathrm{PaCO}_{2}$ : partial pressure of carbon dioxide; $\mathrm{HCO}_{3}-$ : concentration of bicarbonate.

blood vessels. Also obtaining an arterial blood sample in children consumes lot of time owing to various factors and that may delay suitable management such as pediatric intensive care unit (PICU) transfer or intubation. ABG only measures oxygenation at a single time point and dynamic changes in $\mathrm{PaO}_{2}$ may widely get misinterpreted.

On the other hand, pulse oximetry is a quick way of measuring $\mathrm{SpO}_{2}$ of peripheral arterial hemoglobin as it is readily available but cannot detect hypercapnia or acidosis. It relies on the fact that oxygenated and deoxygenated hemoglobin absorb different wavelengths of light. Although ABG analysis remains the gold standard, pulse oximetry assessment has been shown to correlate with ABG analysis results and proposed as a safer less invasive, less painful alternative in children. Aim of this study was to gather evidence by comparison and evaluate $\mathrm{SpO}_{2}$ after administering oxygen through various oxygen delivery patterns with simultaneous pulse oximetry and $\mathrm{ABG}$ analysis to find correlation among them, if any.

\section{Materials and Methods}

In a prospective cross-sectional cohort design adopted for study 60 children from birth to 6 years of age admitted in acute pediatric wards of a single academic tertiary referral hospital in rural setting over a period of 4 months were studied using a validated checklist. Children were eligible for enrollment, if the child is simultaneously put on pulse oximetry monitoring with oxygen support by any oxygen delivery device and the treating pediatricians ordered at least two ABG tests on consecutive days. These children were followed up till they were independent of oxygen supply or for 5 days after admission. Children who were alive at the exit of study only were included for final data in order to have a homogenous group. Children with congenital anomalies or known impaired peripheral perfusion disorders were excluded. This study has been approved by the institutional research/ethical committee.

Pulse oximeter used was 2060 model (EMCO Meditek Pvt. Ltd, Mumbai, India) calibrated for accuracy of reading. Structured observational checklist was prepared that collected brief identification profile, clinical features and investigative profile of child including results of $A B G$ analysis that used the Respiratory Opposite Metabolic Equal (ROME) method for evaluation. After obtaining $\mathrm{pH}$ value, respiratory and metabolic components were studied. If the $\mathrm{pH}$ was abnormal, it was determined whether the respiratory value $\left(\mathrm{PaCO}_{2}\right)$ and meta- bolic value $\left(\mathrm{HCO}_{3}^{-}\right)$are consistent with $\mathrm{pH}$ value to determine whether any compensation is present using the following interpretation table prepared (Table 1) [5]. Interpretation was limited to clinical pictures connected to oxygenation status and its related components and did not divulge into its etiological trajectories.

\section{Results}

\section{Basic demography profile of samples}

Children in majority were infants $(62 \%)$, of second birth order $(53 \%)$, male in gender $(55 \%)$, from rural area $(53 \%)$ and had nuclear type of family predominance (63\%). Among them $57 \%$ had two children in family, $52 \%$ of fathers and $50 \%$ of mothers were graduates, majority of whom (57\% fathers, $38 \%$ mothers) were employed in private firms followed by agriculture as an occupation (17\% fathers and 37\% mothers). Parents were between 25 to 30 years old (62\% fathers and 65\% mothers).

\section{Oxygen delivery patterns and $\mathrm{SpO}_{2}$ levels using pulse oxi- metry}

On admission $73 \%$ of children received oxygen by oxy hood at the rate of 6 to $10 \mathrm{~L}$ per min that sustained $\mathrm{SpO}_{2}>90 \%$ (Table 2). They required oxygen supply for 4 days when $97 \%$ of samples' $\mathrm{SpO}_{2}$ was $>90 \%$ using pulse oximetry. Those who received oxygen by nasal prongs on admission were $11 \%$ but had to be shifted to face mask on day 2 owing to discomfort, restlessness and difficulty in keeping the prongs in situ position. Both face mask and oxy hood at standard rates given were continued up to maximum of 4 days since admission when children improved $(60 \%)$ and were not dependent on oxygen delivery on day 5 .

It was also observed that $88 \%$ children who had $>90 \%$ $\mathrm{SpO}_{2}$ on the day of admission had decreased $\mathrm{SpO}_{2}$ on day 2 after which they improved on day 5 without oxygen. Both oxy hood box and face mask were equally effective at the rates standardized for oxygen delivery to sustain $>90 \% \mathrm{SpO}_{2}$. However, oxy hood was used among $75 \%$ of children on oxygen on day 4 than face mask. It may be attributed to the samples being infants in majority that endorses preference for oxy hood box pattern of oxygen delivery for younger children. 
Table 2. Description of Oxygen Delivery Patterns with $\mathrm{SpO}_{2}$ and $\mathrm{PaO}_{2}$ Levels

\begin{tabular}{|c|c|c|c|c|c|c|c|c|}
\hline \multirow{2}{*}{ Variables } & \multicolumn{2}{|c|}{ Admission $(n=60)$} & \multicolumn{2}{|c|}{ Day $2(n=60)$} & \multicolumn{2}{|c|}{ Day $3(n=60)$} & \multicolumn{2}{|c|}{ Day $4(n=36)$} \\
\hline & n & $\%$ & $\mathbf{n}$ & $\%$ & $\mathbf{n}$ & $\%$ & $\mathbf{n}$ & $\%$ \\
\hline \multicolumn{9}{|l|}{$\mathrm{SpO}_{2}(\%)$} \\
\hline$>90$ & 53 & 88 & 47 & 78 & 55 & 91 & 35 & 97 \\
\hline \multicolumn{9}{|c|}{ Delivery Devices (delivery/min) } \\
\hline Face mask $(5-6)$ & 10 & 16 & 30 & 50 & 30 & 50 & 09 & 25 \\
\hline Oxy hood box $(6-10)$ & 44 & 73 & 30 & 50 & 30 & 50 & 27 & 75 \\
\hline $\mathrm{PaO}_{2}(\mathrm{~mm} \mathrm{Hg})$ & \multicolumn{2}{|c|}{$\mathrm{n}=60$} & \multicolumn{2}{|c|}{$\mathrm{n}=60$} & \multicolumn{2}{|c|}{$\mathrm{n}=41$} & \multicolumn{2}{|c|}{$\mathrm{n}=12$} \\
\hline$<80$ & 11 & 18 & 17 & 28 & 6 & 15 & 2 & 17 \\
\hline $80-100$ & 49 & 82 & 43 & 72 & 35 & 85 & 10 & 83 \\
\hline
\end{tabular}

$\mathrm{SpO}_{2}$ : oxygen saturation; $\mathrm{PaO}_{2}$ : partial pressure of oxygen.

\section{Oxygen delivery patterns and $\mathrm{PaO}_{2}$ using $\mathrm{ABG}$ analysis}

On admission $82 \%$ had $\mathrm{PaO}_{2}$ in the normal acceptable range which is slightly lower than the $\mathrm{SpO}_{2}$ findings by pulse oximeter (Table 2). Other $18 \%$ had $\mathrm{PaO}_{2}$ of $<80 \mathrm{~mm} \mathrm{Hg}$ that increased to $28 \%$ on day 2 which improved to $15-17 \%$ on day 3 and 4 respectively. This trend is comparable with inputs from $\mathrm{SpO}_{2}$ observations used simultaneously.

\section{ABG analysis report of samples}

ABG analysis report interpretation (Fig. 1) found that $35 \%$ had respiratory acidosis, $8 \%$ had respiratory alkalosis, $42 \%$ had metabolic acidosis, 13\% had metabolic alkalosis and 2\% were normal on admission. Consecutive analysis report findings noted in Table 2 with specific focus to $\mathrm{SpO}_{2}$ and $\mathrm{PaO}_{2}$ substantiates hypothesis that support correlation between both the levels. It is further observed that $\mathrm{O}_{2}$ saturation varied with $\mathrm{PaO}_{2}$ in a nonlinear relationship as $\mathrm{PaCO}_{2}$ was not affected initially on admission but the disturbance was evident on day 2 supporting the notion that the diffusion capacity of $\mathrm{CO}_{2}$ is much higher than that of oxygen and only when alveolar level ventilation is

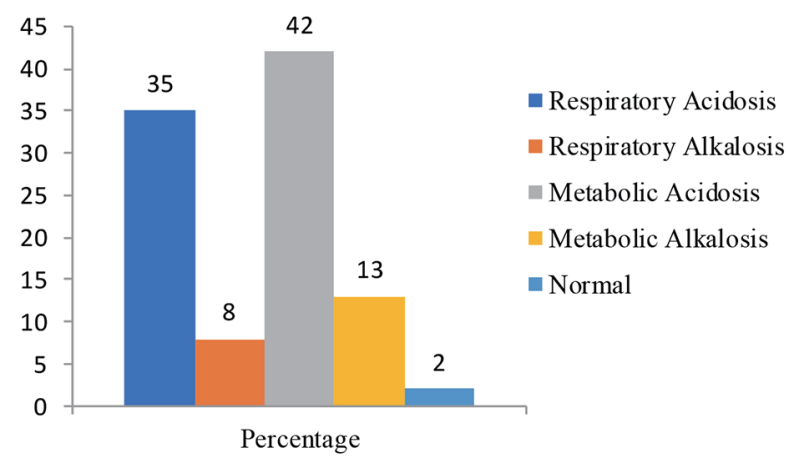

Figure 1. $A B G$ analysis report interpretation of children. $A B G$ : arterial blood gas. impaired its values get affected. The number of ABGs ordered per sample was limited to a maximum of two in $32 \%$, three in $68 \%$ and four in $20 \%$ of children, who were critically ill and thus a cascade of reports were also restricted as days advanced.

As shown in Table 3 on admission majority of children with respiratory acidosis manifested with altered sensorium (44\%), respiratory alkalosis with restlessness (84\%) and hyperventilation, metabolic acidosis with decreased cardiac function (79\%) and Kussmaul breathing, metabolic alkalosis with refusal to feeds $(50 \%)$ and irritability (64\%) and all four disturbances had children presenting with unspecific features in varying proportions mentioned. Treatment regimen offered to children under $\mathrm{SpO}_{2}$ and $\mathrm{PaO}_{2}$ monitoring were oxygen administration $(100 \%)$, ventilation with bilevel positive airway pressure (BiPAP) and continuous positive airway pressure (CPAP) (62\%), antibiotics (100\%), $\mathrm{K}^{+}$supplements (27\%), sodium bicarbonate $\left(\mathrm{NaHCO}_{3}\right)$ supplements $(32 \%)$ and correction of primary problems using medications, intravenous (IV) fluids plus supportive nursing care (100\%).

Correlation coefficient test was used to evaluate correlation between $\mathrm{SpO}_{2}$ and oxygen delivery patterns that obtained $r$ value of 0.8 , which interprets that $\mathrm{SpO}_{2}$ had positive correlation with oxygen delivery methods. Same test used to evaluate correlation between $\mathrm{PaO}_{2}$ and $\mathrm{SpO}_{2}$ obtained $\mathrm{r}$ value of 0.9 that it interprets positive correlation between $\mathrm{PaO}_{2}$ had with $\mathrm{SpO}_{2}$.

\section{Discussion}

Supplemental oxygen in pediatrics poses challenges owing to wide range of size in choosing an appropriate oxygen delivery pattern to meet dynamic physiological instability. ABG analysis and pulse oximetry are two types of measurements that provide insight into oxygenation levels in child receiving continuous ventilation support. Pulse oximeter is an important monitoring tool that allows decisions on constant titration of oxygen supply dose, early diagnosis of desaturation upon which several studies have debated its benefits and disadvantages for over two decades and more. However, the confidence 


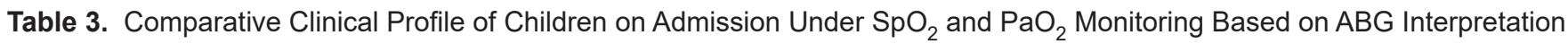

\begin{tabular}{|c|c|c|c|c|c|c|c|}
\hline Respiratory acidosis $(n=21)$ & $\%$ & $\begin{array}{l}\text { Respiratory } \\
\text { alkalosis }(n=5)\end{array}$ & $\%$ & $\begin{array}{l}\text { Metabolic aci- } \\
\text { dosis }(n=25)\end{array}$ & $\%$ & $\begin{array}{l}\text { Metabolic alka- } \\
\text { losis }(n=8)\end{array}$ & $\%$ \\
\hline Shortness of breath & 33 & Hypokalemia & 21 & Vomiting & 63 & Increased HR & 36 \\
\hline Increased HR & 28 & Hyperventilation & 57 & Kussmaul breathing & 74 & Refusal to feeds & 50 \\
\hline Dysrhythmia & 16 & Seizure & 2 & Dec cardiac function & 79 & Restlessness/irritability & 64 \\
\hline Unspecific & 56 & & & & & & \\
\hline
\end{tabular}

ABG: arterial blood gas; $\mathrm{SpO}_{2}$ : oxygen saturation; $\mathrm{PaO}_{2}$ : partial pressure of oxygen; HR: heart rate.

quotient on its extensive use at critical/peripheral pediatric care practice is with hesitancy resulting its use as an adjunct monitoring device and instead, trends of sequential ABG tests remain as the mainstay in treatment decisions. This study compared commonly used oxygen delivery patterns and evaluated its impact on monitoring simultaneously with both methods of $\mathrm{SpO}_{2}$ with its correlation.

Infants age group received oxygen supply more commonly among children which is noted by Ramachandran et al [7] (75\%), Kumar et al [6] (68\%), similar to the finding in this study $(62 \%)$. It could be because that they are the commonest pediatric age group who are affected by respiratory disorders. Male predominance of 55\% among samples is also observed by other studies - Tiwari et al [8] (52\%), LeCleir et al [9] (57\%) and Ramachandran et al [7] (73\%), all of which have not shown any statistical significance.

Requirement of oxygen supply for an average period of 4 days (96 h) is shared by LeCleir et al [9] and Bhargava et al [10]. This clinical variable is significant as it decides the length of their stay (LOS) and course in hospital. It is possible that due to a true presentation with illness severity which children reach tertiary care centers mandates lengthy $\mathrm{O}_{2}$ delivery. Oxy hood and face mask as an effective and well tolerated method for oxygen administration in children has been concluded by Kumar et al [6] (70\%) using a tolerance score, in synchrony as this study ( $73 \%$ on admission to $50 \%$ subsequently).

There was no significant difference among two high-flow methods (face mask and head box) to maintain oxygenation and saturation. Yet the difference between high-flow and lowflow methods (nasal prongs in this study and nasal catheter in other studies) in their ability to relieve hypoxemia was highly significant $(\mathrm{P}=0.001$ and $\mathrm{P}=0.030$, respectively). This may be attributed to the pain and discomfort caused to the fragile skin with constant pressure exerted on nose, cheeks and tapes used on face as a precaution to secure them. However, Walsh et al [2] opines otherwise in their review on these devices in view of the cost involved during hospitalization.

Pulse oximetry has been evaluated in several studies [6, $7,10-14]$ as a reliable tool that has shown positive results in measuring $\mathrm{SpO}_{2}$ with equal accuracy in most scenarios in comparison to $\mathrm{ABG}$ analysis. A correlation between $\mathrm{SpO}_{2}$ and $\mathrm{PaO}_{2}$ was proposed [11] with a simple hypothesis that if the monitor is showing a $\mathrm{SpO}_{2}$ of $70 \%$, we can almost consider a value of $\mathrm{PaO}_{2}$ to be around $35 \mathrm{~mm} \mathrm{Hg}$. Use of noninvasive indices $\left(\mathrm{SpO}_{2}\right)$ was suggested [10] in place of invasive indices $\left(\mathrm{PaO}_{2}\right)$ as surrogate markers of oxygenation in critically ill children. Another study [7] observed that pulse oximeter detected $\mathrm{SpO}_{2}$ comparable to arterial $\mathrm{SpO}_{2}$ in all the patients with cyanotic heart diseases, even though most of them did not present with clinically significant cyanosis. This study concurred with above findings as well with other studies [12, 13] which found that $\mathrm{PaO}_{2}$ had positive correlation with $\mathrm{SpO}_{2}$ and noted that readings of $\mathrm{SpO}_{2}$ of $>90 \%$ correlated with $\mathrm{PaO}_{2}$ of $>80 \mathrm{~mm} \mathrm{Hg}$ for $>94 \%$ of the time with statistically significant coefficient values of $\mathrm{r}=0.8$ with oxygen delivery patterns and $\mathrm{r}=0.9$ with simultaneous $\mathrm{SpO}_{2}$ and $\mathrm{PaO}_{2}$ recordings.

Moreover, it was observed clinically that a pulse oximeter provided measurements of heart rate as an additional vital sign aided initiation of appropriate care measures for child. A study [15] on the contrary cautioned against surrogate use of $\mathrm{SpO}_{2}$ for $\mathrm{PaO}_{2}$. Reason could be illustrated with the principle of differential absorption of light by oxygenated and deoxygenated blood where increased carboxyhemoglobin levels are independently associated with the percentage difference between $\mathrm{SpO}_{2}$ and $\mathrm{PaO}_{2}$ and hence pulse oximetry does not provide insight on the level of $\mathrm{PaCO}_{2}$ or $\mathrm{pH}$. However, simultaneous pulse oximetry measurements accurately projected oxygenation status in children undergoing various oxygen delivery patterns with significant statistical correlation of arterial saturation measured by ABG analysis in this study. The rapid response time of $\mathrm{SpO}_{2}$ offered prompt and important decisions at bedside guiding treatment regarding oxygen therapy.

ABG analysis reports highlighted comparable acid base disturbances with $42 \%$ samples having metabolic acidosis similar to study samples by Anitha et al [16] (47\%), which commonly occurs in critically ill children [17]. BiPAP and CPAP had been found as the best ventilation mode for $78 \%$ participants to treat respiratory disorders in consistency with prescription by Abramo et al [18]. $\mathrm{NaHCO}_{3}$ supplementation remained as the mainstay in treating acid base disorders, a common clinical practice noted by Daly et al [19].

\section{Conclusions}

Oxygen delivery patterns by oxy hood and face mask have bet- 
ter tolerance and comfort among infants with an effective impact to maintain $\mathrm{SpO}_{2}$. There is a positive correlation between oxygen delivery methods with $\mathrm{PaO}_{2}$ and $\mathrm{SpO}_{2}$. Pulse oximetry can be used as a reliable bed side tool to evaluate effectiveness of oxygen delivery patterns and diagnose early respiratory failure superior to $\mathrm{ABG}$ in emergency, peripheral and rural healthcare settings where ABG analysis facility is not available and cost is a considerable factor.

\section{Acknowledgments}

The authors would like to thank Prarvara Institute of Medical Sciences (Deemed to be University) and Pravara Rural Hospital Loni authorities for all.

\section{Financial Disclosure}

None to declare.

\section{Conflict of Interest}

None to declare.

\section{Informed Consent}

The informed consents have been obtained from the parents.

\section{Author Contributions}

DG: Literature exploration, research data collection, statistical analysis and first draft. LVR: Guide, concept, research design, literature exploration and final draft. VB: Research guidance, clinical support, co-ordination.

\section{Data Availability}

The data supporting the findings of this study are available from the corresponding author upon reasonable request.

\section{References}

1. Frey B, Shann F. Oxygen administration in infants. Arch Dis Child Fetal Neonatal Ed. 2003;88(2):F84-88.

2. Walsh BK, Smallwood CD. Pediatric oxygen therapy: a review and update. Respir Care. 2017;62(6):645-661.

3. Soini UM, Clarke S, Solberg MT. Oxygen treatment of critically ill children: A lack of evidence. Nordic Journal of Nursing Research. 2016;37(3):127-134.

4. Hasan A. The non-invasive monitoring of blood oxygen and Carbon dioxide levels. Handbook of blood gas/acid base Interpretation. 2013:51-93.
5. Larkin BG, Zimmanck RJ. Interpreting arterial blood gases successfully. AORN J. 2015;102(4):343-354; quiz 355-347.

6. Kumar RM, Kabra SK, Singh M. Efficacy and acceptability of different modes of oxygen administration in children: implications for a community hospital. J Trop Pediatr. 1997;43(1):47-49.

7. Ramachandran S, Gera A, Gera R, Kataria S, Roy MP. Accuracy of pulse oximetry versus arterial blood gas in screening cyanotic heart. International Journal of Contemporary Pediatrics. 2016;3(3):983-987.

8. Tiwari S, Soumya T, Nangia S, Saili A. Oxygen saturation profile in healthy term neonates in the immediate postnatal period. Int J Clin Pediatr. 2013;2(1):19-23.

9. LeCleir B, Jurecko L, Davis AT, et al. Implementing an oxygen supplementation and monitoring protocol on inpatient pediatric Bronchiolitis: An exercise in de-implementation. Int J Pediatr. 2017;3169098:1-7.

10. Bhargava S, Pooni P, Singla S. Comparison of $\mathrm{SpO} 2$ and $\mathrm{PaO} 2$ based indices of oxygenation in children with respiratory failure. Pediatric Critical Care Medicine. 2018;19(6S):120.

11. Madan A. Correlation between the levels of $\mathrm{SpO} 2$ and PaO2. Lung India. 2017;34(3):307-308.

12. Ramy R, El-Sberbihy, Ahmed A, El-Ezz A, Ibrahim, Yasser M, El-raouf A, et al. Pulse oximetry in comparison to arterial blood oxygen saturation in children with bronchial asthma coming to the emergency room. Tanta Medical Journal. 2018;46(2):93-98.

13. Ross EM, Matteucci MJ, Shepherd M, Barker M, Orr L. Measuring arterial oxygenation in a high altitude field environment: comparing portable pulse oximetry with blood gas analysis. Wilderness Environ Med. 2013;24(2):112117.

14. Fanconi S, Doherty P, Edmonds JF, Barker GA, Bohn DJ. Pulse oximetry in pediatric intensive care: comparison with measured saturations and transcutaneous oxygen tension. J Pediatr. 1985;107(3):362-366.

15. Bhattacharyya A, Ramos J, Tonelli AR. Discrepancy between pulse oximetry and arterial oxygen saturation at rest and during exercise. American Journal of Respiratory and Critical Care Medicine. 2020;201:A5451. Available at: https://www.atsjournals.org/doi/abs/10.1164/ajrccmconference.2017.195.1.

16. Anitha B, Siromani SSM. Acid base disturbances in sick neonates - An observational study in a tertiary care centre. IOSR Journal of Dental and Medical Sciences. 2015;14(12):35-38.

17. Sekaran DV, Subramanyam L, Balachandran A. Arterial blood gas analysis in clinical practice. Indian Pediatr. 2001;38(10):1116-1128.

18. Abramo T, Williams A, Mushtaq S, Meredith M, Sepaule R, Crossman K, Burney Jones C, et al. Paediatric ED BiPAP continuous quality improvement programme with patient analysis: 2005-2013. BMJ Open. 2017;7(1):e011845.

19. Daly K, Farrington E. Hypokalemia and hyperkalemia in infants and children: pathophysiology and treatment. J Pediatr Health Care. 2013;27(6):486-496; quiz 497-488. 